\title{
Magnetic Properties and Magnetic Domain Observation of Nd-Fe-B Sintered Magnets Treated by Grain Boundary Diffusion Process with Dy-Al Co-Sorption
}

\author{
Masaru Uenohara ${ }^{1,3, *}$, Hiroaki Nishio ${ }^{2,4}$, Kentaro Toyoki ${ }^{1}$, Yu Shiratsuchi ${ }^{1}$, Ryoichi Nakatani ${ }^{1}$ and \\ Ken-ichi Machida ${ }^{2}$ \\ ${ }^{1}$ Department of Materials Science and Engineering, Graduate School of Engineering, Osaka University, Suita 565-0871, Japan \\ ${ }^{2}$ Department of Applied Chemistry, Graduate School of Engineering, Osaka University, Suita 565-0871, Japan \\ ${ }^{3}$ Division of Powertrain Production Engineering, Nissan Motor Company, Yokohama 230-0053, Japan \\ ${ }^{4}$ Research Institute for Measurement of Magnetic Materials, Yokohama 240-0026, Japan
}

Effective grain boundary diffusion (GBD) process with Dy-Al co-sorption is applied to enhance the coercivity of Nd-Fe-B sintered magnets. The coercivity of the magnet $\left(H_{c J}=1789 \mathrm{kA} \cdot \mathrm{m}^{-1}\right)$ subjected to the present GBD treatment was observed to be superior to that of the untreated magnet $\left(H_{c J}=1003 \mathrm{kA} \cdot \mathrm{m}^{-1}\right)$ and the conventional GBD magnet $\left(H_{c J}=1661 \mathrm{kA} \cdot \mathrm{m}^{-1}\right)$ treated with DyAl alloy. In the present GBD magnet, the Dy-Al co-sorption process facilitated Dy diffusion into the center region of the magnet (thickness: $3.5 \mathrm{~mm}$ ), resulting in high coercivity. Further, magnetic domain observations were made using magnetic force microscopy (MFM) to observe the thermal demagnetization behavior of the present GBD magnet. The present GBD magnet suppressed the continuous domain reversal of adjacent grains; thus, the partially persistent single-domain structure remained, even at 453 K. [doi:10.2320/matertrans.MT-M2020389]

(Received December 25, 2020; Accepted April 30, 2021; Published June 18, 2021)

Keywords: neodymium-iron-boron magnets, coercivity, grain boundary diffusion

\section{Introduction}

The addition of heavy rare-earth $(\mathrm{HRE}=\mathrm{Dy}$ or $\mathrm{Tb})$ elements in $\mathrm{Nd}-\mathrm{Fe}-\mathrm{B}$ sintered magnets efficiently enhances the coercivity $\left(H_{c J}\right)^{1-3)}$ but reduces the maximum energy product, $(B H)_{M a x}$, because of the reduction in the remanence $\left(B_{r}\right)$ due to the antiferromagnetic coupling between the HRE elements and $\mathrm{Fe}$ in the $(\mathrm{Nd}, \mathrm{Dy})_{2} \mathrm{Fe}_{14} \mathrm{~B}$ main phase. Recently, the price of these sintered magnets has been increasing because of insufficient HRE resources, which is a major problem in the industrial field. Furthermore, given the increasing awareness of environmental issues, magnets with high coercivity, particularly those applicable in electric vehicles $(\mathrm{EV} / \mathrm{HEV})$, are in high demand. ${ }^{4)}$ Therefore, it is necessary to improve the coercivity of $\mathrm{Nd}-\mathrm{Fe}-\mathrm{B}$ sintered magnets while minimizing the amount of HRE used.

The process of selectively enriching the HRE elements in the grain boundary region surrounding the $\mathrm{Nd}_{2} \mathrm{Fe}_{14} \mathrm{~B}$ main phase, that is, the core-shell type microstructure, is an efficient way to reduce the amount of HRE in the $\mathrm{Nd}-\mathrm{Fe}-\mathrm{B}$ sintered magnets. To obtain a suitable core-shell type microstructure, a grain boundary diffusion (GBD) process in which the magnets were coated by HRE elements and subsequently heated above the melting point of the Nd-rich grain boundary was developed. ${ }^{5-18)}$ Following heat treatment, the resulting magnets exhibit high coercivity and suppress the decrease in remanence.

We previously reported a GBD process in which Dy or $\mathrm{Tb}$ metals were coated on the magnet surface by a sputtering method $^{7)}$ or a reduction diffusion method applied to HRE compounds and $\mathrm{Ca}$ reductants. ${ }^{8,17)}$ Using these techniques, a structure in which the $\mathrm{Nd}_{2} \mathrm{Fe}_{14} \mathrm{~B}$ main phase is surrounded by the HRE-enriched grain boundary was achieved. Consequently, the coercivity was effectively enhanced without a severe reduction in the remanence. We also reported

*Corresponding author, E-mail: m-uenohara@mail.nissan.co.jp improved magnetic properties of $\mathrm{Nd}-\mathrm{Fe}-\mathrm{B}$ scrap powder by $\mathrm{Yb}$ metal sorption (both adsorption and absorption), utilizing the low boiling point of the HRE elements. ${ }^{9)}$ Similar GBD processes using various HRE compound powders, ${ }^{10-13)}$ vapor deposition, ${ }^{5,6,14-16)}$ and alloy powder ${ }^{18,19)}$ have also been reported. In particular, a GBD process using $\mathrm{Dy}-\mathrm{Ni}-\mathrm{Al}$ alloy powder was reported by Oono et al.; ${ }^{18)}$ the simultaneous Dy diffusion with $\mathrm{Ni}$ and $\mathrm{Al}$ yielded a decrease in the melting point of the grain boundary. As a result, Dy diffusion into the deeper region of the magnets was facilitated, resulting in high coercivity, even with a thickness of $5 \mathrm{~mm}$. However, the preparation of the alloy powder requires largescale equipment for melting and grinding the alloy, and the fine alloy powder containing HRE elements is extremely active in air atmosphere; thus, handling and mass productivity become a problem.

Meanwhile, in the GBD process, the HRE elements diffuse from the magnet surface, causing a difference in the HRE concentration near the surface and deep regions in the magnets, which limits both the applicable magnet size and the increase in coercivity. In mass production, to achieve high coercivity, the GBD process with $\mathrm{Tb}$ raw material, which has a higher magnetic anisotropy and is more expensive than Dy, is widely applied.

Referring to a previous study, ${ }^{18)}$ to obtain a more effective coercivity enhancement of $\mathrm{Nd}-\mathrm{Fe}-\mathrm{B}$ sintered magnets using the GBD process with Dy, which is cheaper than $\mathrm{Tb}$, this study focuses on the melting point of the grain boundary and investigates the simple GBD process with Dy-Al co-sorption. Dy (b.p. $=2835 \mathrm{~K}^{20)}$ ) and $\mathrm{Al}$ (b.p. $=2740 \mathrm{~K}^{21)}$ ) are lowboiling-point elements, and sorption readily occurs under reduced pressure conditions such as $1.0 \times 10^{-3} \mathrm{~Pa}^{22)}$ Conventionally, $\mathrm{Al}$ has been used as a minor additive element in Nd-Fe-B sintered magnets. ${ }^{23,24)}$ The diffusion of Al into the Nd-rich grain boundary yields a decrease in the melting point, ${ }^{25)}$ and Dy diffusion into the deeper region of the magnet is expected. 
Although there are numerous reports on the grain boundary microstructure of GBD magnets, ${ }^{5,6,14,15)}$ there are several reports on the thermal demagnetization behavior at high temperatures, which are important properties for the driving motors of electric vehicles. Therefore, to investigate the demagnetization behavior in more detail, magnetic domain observations using magnetic force microscopy (MFM) were also performed to observe the thermal demagnetization behavior of the present GBD magnet treated by $\mathrm{Dy}-\mathrm{Al}$ co-sorption.

\section{Experimental}

In this study, the conventional and present GBD processes were applied. The conventional GBD process applied Dy sorption, DyAl alloy, and a mixture of $\mathrm{TbF}_{3}$ and $\mathrm{CaH}_{2}$. The procedure for Dy sorption and a mixture of $\mathrm{TbF}_{3}$ and $\mathrm{CaH}_{2}$ were based on previous reports. ${ }^{8,16)}$ The DyAl alloy with a molar ratio of $\mathrm{Dy}: \mathrm{Al}=1: 1$ was prepared by arc melting, grinding to approximately $5 \mu \mathrm{m}$, then coating onto the magnet surface via anhydrous-butanol. The present GBD process applied Dy-Al co-sorption. Figure 1 shows a typical experimental procedure for the present GBD process. Massproduced $\mathrm{Nd}-\mathrm{Fe}-\mathrm{B}$ sintered magnets $\left(B_{r}=1.43 \mathrm{~T}, H_{c J}=\right.$ $\left.1003 \mathrm{kA} \cdot \mathrm{m}^{-1}\right)$ were cut into square-shaped $(3.0 \times 3.0 \times$ $\left.2.8 \mathrm{~mm}^{3}\right)$ or cylindrical $\left(10^{\phi} \times 3.5^{\mathrm{t}} \mathrm{mm}^{3}\right)$ pieces by machining. The oxide layer on the magnet surface was subsequently removed by etching with dilute nitric acid for several minutes. Al metal powder ( $99.0 \%$ purity) with an average particle diameter of approximately $30 \mu \mathrm{m}$ and anhydrousbutanol were mixed at a weight ratio of 50:50. After immersing the magnets in this slurry solution, hot air drying was performed to uniformly coat the $\mathrm{Al}$ powder on the magnet surface. The Al-coated magnet was vacuum-sealed with Dy metal grains (purity 99.9\%) in a quartz tube maintained at a vacuum degree of $1.0 \times 10^{-3} \mathrm{~Pa}$. The quartz tubes were heated at $1173-1273 \mathrm{~K}$ for $6 \mathrm{hrs}$ and subsequently annealed at $823 \mathrm{~K}$ for $2 \mathrm{hrs}$. After heat treatment, the re-

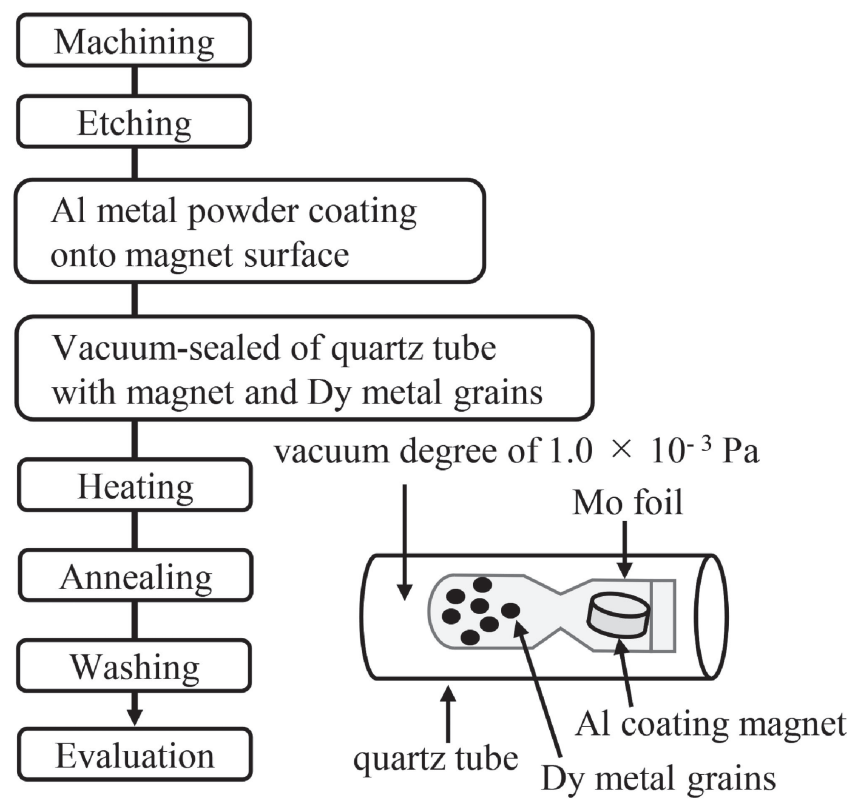

Fig. 1 Typical experimental procedure for the present GBD process. sulting GBD magnets were ultrasonically washed with dilute nitric acid and ethanol to remove impurities on the magnet surface.

The magnetic properties were evaluated using a pulseexcitation-type $\mathrm{BH}(\mathrm{PBH})$ tracer or a superconducting magnet-based vibrating sample magnetometer (SCM-VSM) with a magnetic field range of $\pm 4775 \mathrm{kA} \cdot \mathrm{m}^{-1}$. The SCMVSM measurement method was described in previous papers. ${ }^{26,27)}$ Microstructural characterizations were performed using an X-ray diffractometer (XRD), field emission electron probe micro-analyzer (FE-EPMA), scanning transmission electron microscope (STEM). The chemical composition was quantitatively analyzed by inductively coupled plasma (ICP) optical emission spectrometry. Magnetic domain observations were performed using MFM. A high coercivity probe with a coercivity of $>796 \mathrm{kA} \cdot \mathrm{m}^{-1}$ was applied. By following the probe based on the pre-scanned shape images, the distance between the magnet surface and the probe can remain constant at approximately $20-30 \mathrm{~nm}$. The MFM observations were performed in situ after increasing the temperature from $300 \mathrm{~K}$ to $373 \mathrm{~K}$ and $453 \mathrm{~K}$ in a vacuum degree of approximately $1.0 \times 10^{-4} \mathrm{~Pa}$ and maintaining it for $1.5 \mathrm{hrs}$ at each predetermined temperature. Before the observation, the magnets were magnetized by a pulsed magnetic field of $6366 \mathrm{kA} \cdot \mathrm{m}^{-1}$ after flat-mill-polishing on the magnet surface. Note that, since the evaluations of the magnetic properties and the MFM observations were performed at different site facilities, the magnetized magnets were vacuum-packed to enable transportation.

\section{Results and Discussion}

\subsection{XRD patterns and magnetic properties}

Figure 2 shows the XRD patterns measured on the magnet surface (a) treated by the present GBD process with Dy-Al co-sorption at $1223 \mathrm{~K}$ for $6 \mathrm{hrs}$ and (b) untreated. Here, the untreated magnet referred to a magnet that was sintered and subsequently annealed. Note that, acid washing or polishing was not performed before XRD. In the present GBD magnet, the peak intensity of the fcc $\mathrm{NdO}$ phase (measured lattice constant: $a=0.5025 \mathrm{~nm}$ ) was clearly increased compared to

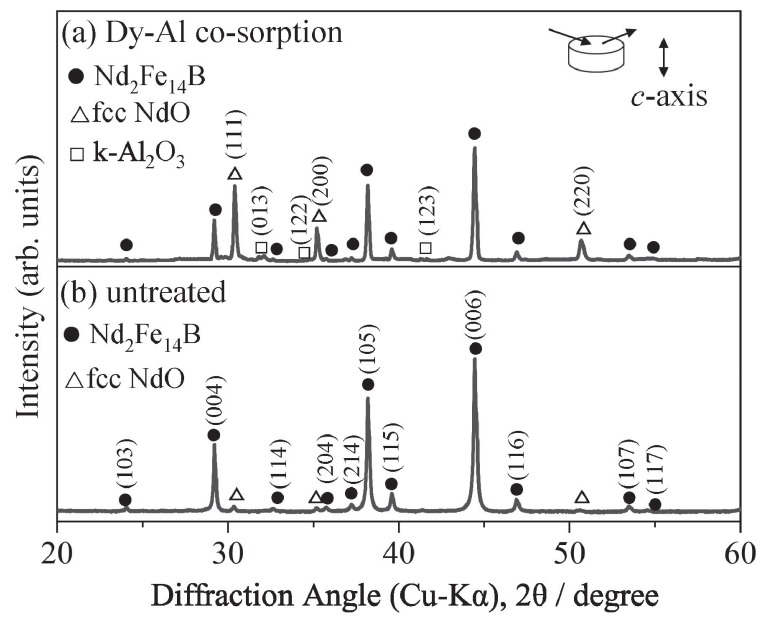

Fig. 2 XRD patterns of the magnet surface (a) treated by the present GBD process with Dy-Al co-sorption at $1223 \mathrm{~K}$ for $6 \mathrm{hrs}$ and (b) untreated. 


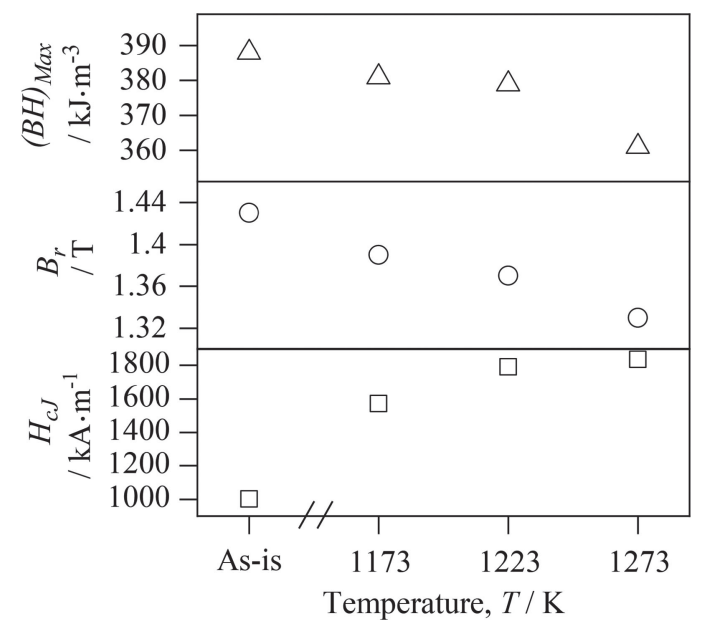

Fig. 3 Dependence of magnetic properties $\left(H_{c J}, B_{r}\right.$, and $\left.(B H)_{M a x}\right)$ on the heat treatment temperature for the present GBD magnet.

the untreated magnet. This indicated that an Nd-rich phase was regenerated on the magnet surface by the GBD process. However, the peak intensity of the fcc $\mathrm{NdO}$ phase was extremely weak in the untreated magnet, that is, the $\mathrm{Nd}_{2} \mathrm{Fe}_{14} \mathrm{~B}$ main phase was almost bare on the magnet surface before the GBD process. This was presumably due to the oxidation of the Nd-rich phase caused by machining or surface oxidation, and subsequently peeled off the magnet surface. Meanwhile, the regeneration of the Nd-rich phase was due to Dy, which was vapor-deposited on the magnet surface and diffused into the inner region by substituting with $\mathrm{Nd}$, which was enriched in the grain boundary. This is similar to the case where $\mathrm{Nd}$ was sputter-deposited on the magnet surface and heat treatment was subsequently applied. ${ }^{28)}$ This result indicated that, like $\mathrm{Nd}$, Dy recovered the magnet surface, which deteriorates owing to oxidation and machining. While, Al powders pre-coated on the magnet surface also became vapor during heat treatment under the present conditions. However, because $\mathrm{Al}$ powders were coated directly on the magnet surface, Al sorption proceeded preferentially compared to Dy sorption.

Figure 3 shows the dependence of magnetic properties $\left(H_{c J}, B_{r}\right.$, and $\left.(B H)_{M a x}\right)$ on the heat treatment temperature for the $3.0 \times 3.0 \times 2.8 \mathrm{~mm}^{3}$ magnets treated by the present GBD process. The heat time was constant at $6 \mathrm{hrs}$, and the values were evaluated by the $\mathrm{PBH}$ tracer. The coercivity $\left(H_{c J}\right)$ increased with increasing heat treatment temperature, and the remanence $\left(B_{r}\right)$ and maximum energy product $\left((B H)_{M a x}\right)$ decreased because of the reduction of the saturation magnetization $\left(M_{S}\right)$ by proceeding with the interstitial diffusion of Dy into the $\mathrm{Nd}_{2} \mathrm{Fe}_{14} \mathrm{~B}$ main phase. In particular, when the heat treatment temperature increased to $1273 \mathrm{~K}$, which is close to the melting point of the $\mathrm{Nd}_{2} \mathrm{Fe}_{14} \mathrm{~B}$ main phase, the coercivity became highly enhanced from $1003 \mathrm{kA} \cdot \mathrm{m}^{-1}$ to $1835 \mathrm{kA} \cdot \mathrm{m}^{-1}$. In addition, even at a heat temperature of $1173 \mathrm{~K}$, the coercivity $\left(H_{C J}=1571 \mathrm{kA} \cdot \mathrm{m}^{-1}\right)$ exceeded the conventional GBD process applying Dy as a diffusion material, such as mixed powder of $\mathrm{DyF}_{3}+\mathrm{CaH}_{2}$ $\left(H_{c J}=1420 \mathrm{kA} \cdot \mathrm{m}^{-1}\right){ }^{8)}$ Based on the results, we can select the heat treatment conditions according to the required coercivity and remanence. In this study, the basic heat

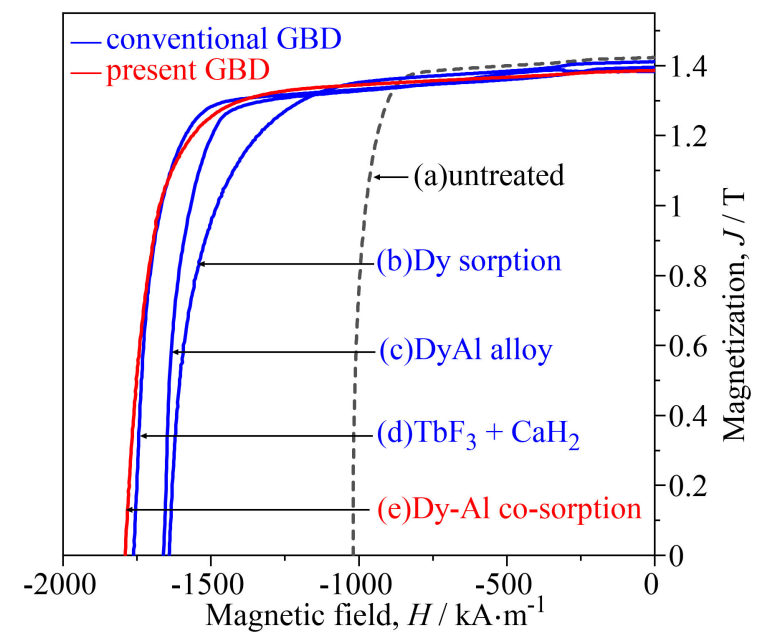

Fig. 4 Demagnetization curves of the magnets measured by SCM-VSM (a) untreated, treated by the conventional GBD process with (b) Dy sorption, (c) DyAl alloy, (d) mixture of $\mathrm{TbF}_{3}$ and $\mathrm{CaH}_{2}$, and (e) treated by the present GBD process with Dy-Al co-sorption. Heat treatment conditions remain constant at $1223 \mathrm{~K}$ for $6 \mathrm{hrs}$.

Table 1 Summary of the magnetic properties of the (a) untreated magnet, (b)-(d) conventional GBD magnets, and (e) the present GBD magnet.

\begin{tabular}{lcccc}
\hline & $\begin{array}{c}H_{c J} \\
/ \mathrm{kA} \cdot \mathrm{m}^{-1}\end{array}$ & $\begin{array}{c}B_{r} \\
/ \mathrm{T}\end{array}$ & $\begin{array}{c}H_{k} / H_{c J} \\
(\%)\end{array}$ & $\begin{array}{c}(\mathrm{BH})_{\operatorname{Max}} \\
/ \mathrm{kJ} \cdot \mathrm{m}^{-3}\end{array}$ \\
\hline (a) untreated & 1003 & 1.43 & 91 & 388 \\
\hline (b) Dy sorption & 1641 & 1.41 & 77 & 378 \\
(c) DyAl alloy & 1661 & 1.39 & 89 & 375 \\
(d) $\mathrm{TbF}_{3}$ and $\mathrm{CaH}_{2}$ & 1761 & 1.40 & 90 & 387 \\
(e) Dy-Al co-sorption & 1789 & 1.38 & 85 & 342 \\
\hline
\end{tabular}

treatment temperature was set to $1223 \mathrm{~K}$ based on the increased coercivity and decreased ratio of remanence.

Figure 4 shows the demagnetization curves of the untreated and treated (conventional and present GBD processes) magnets evaluated by SCM-VSM. The heat treatment conditions were constant at $1223 \mathrm{~K}$ for $6 \mathrm{hrs}$ and subsequently annealed at $823 \mathrm{~K}$ for $2 \mathrm{hrs} . H_{c J}, B_{r}, H_{k} / H_{c J}$, and $(B H)_{M a x}$ are summarized in Table 1 . Here, $H_{k}$ is the value of the magnetic field required to reduce the remanence $\left(B_{r}\right)$ by $10 \%$. The magnets were cylindrical, with dimensions of $10^{\phi} \times 3.5^{\mathrm{t}} \mathrm{mm}^{3}$. Independent of the details of the GBD process, the coercivity was enhanced while suppressing the reduced remanence. This demonstrated that GBD process is a suitable process for manufacturing high-coercivity magnets. The present GBD magnet exhibited the highest coercivity among the studied magnets. This increase in coercivity is the same as that of the conventional GBD process with a mixed powder of $\mathrm{TbF}_{3}$ and $\mathrm{CaH}_{2}$; that is, the present GBD process is effective for the substitution of $\mathrm{Tb}$ by Dy. Interestingly, the increase in coercivity was higher than that of the DyAl alloy containing $\mathrm{Al}$ as a diffusion material, as well as Dy-Al cosorption. The reasons for this are discussed in the microstructure section. On the other hand, the GBD process with uniformly coated powders on the magnet surface with the (c) DyAl alloy or (d) $\mathrm{TbF}_{3}$ and $\mathrm{CaH}_{2}$ yielded a higher squareness 


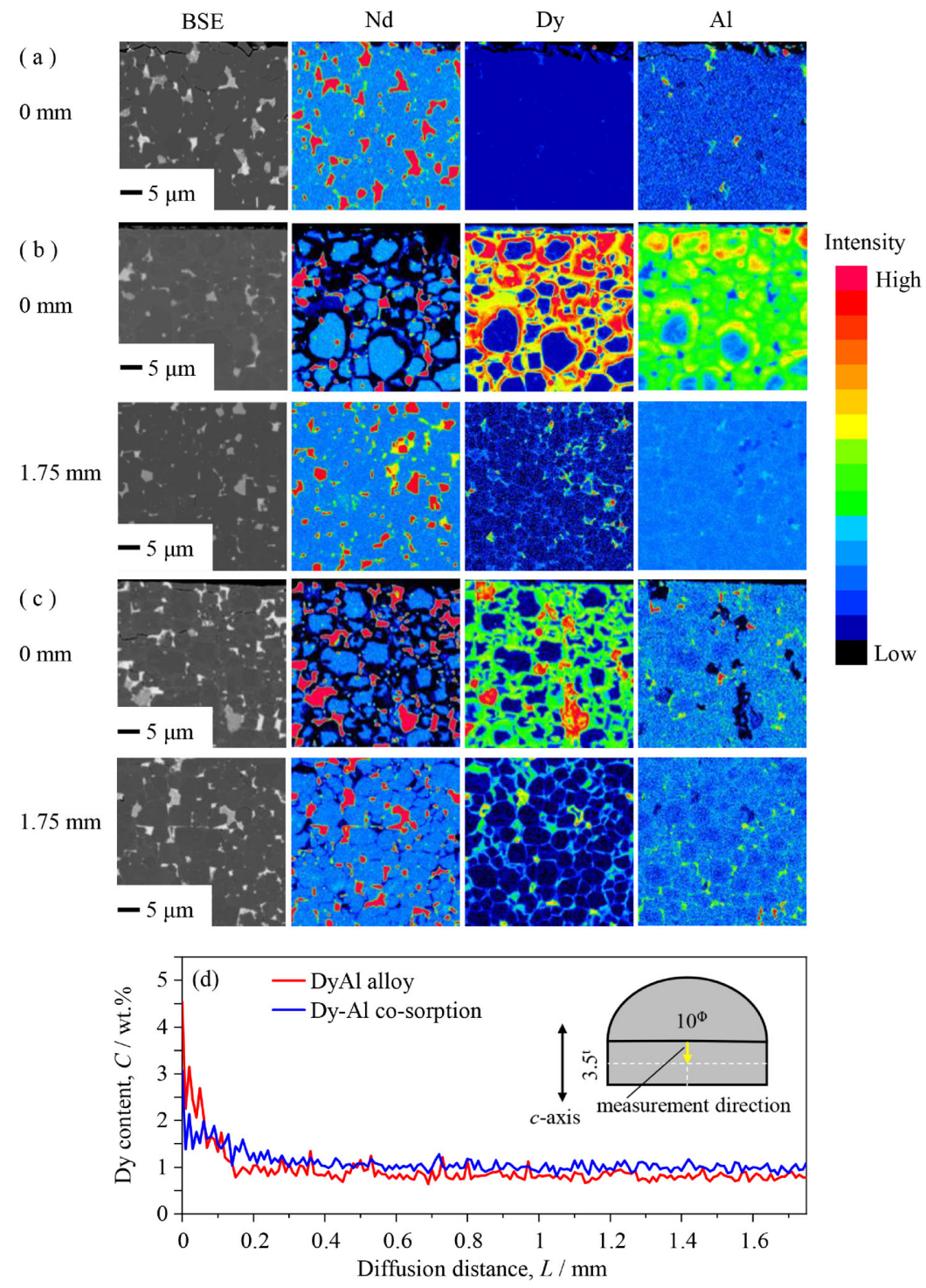

Fig. 5 BSE images and EPMA images of Nd, Dy, and $\mathrm{Al}$ at various depths from the magnet surface (a) untreated, (b) treated by the conventional GBD process with DyAl alloy and (c) treated by the present GBD process with Dy-Al co-sorption. (d) Dy line profile of each GBD magnet obtained by EPMA line analysis.

$\left(H_{k} / H_{c J}\right)$ than the GBD process including Dy sorption (b) and (e). This is because of the uniform coating of HREcontaining powders on the magnet surface, which enabled more uniform diffusion of HRE into the magnets.

\subsection{Observation of grain boundary microstructure}

Figure 5 shows the backscattered electron (BSE) images and EPMA images of $\mathrm{Nd}$, Dy, and $\mathrm{Al}$ at various depths from the untreated and treated (conventional GBD process with DyAl alloy and present GBD process with Dy-Al co-sorption) magnet surfaces, and the Dy line profile of each GBD magnet obtained by EPMA line analysis. The compositions of $\mathrm{Nd}$, Dy, and $\mathrm{Al}$ for each magnet quantitatively analyzed by ICP are summarized in Table 2 . In each GBD magnet, Dy was excessively enriched around the $\mathrm{Nd}_{2} \mathrm{Fe}_{14} \mathrm{~B}$ main phase near the magnet surface, particularly in the case of the conventional GBD process with DyAl alloy, the Dy diffusion into the main phase was remarkable (see Fig. 5(b) and (c) $0 \mathrm{~mm}$-Dy). On the other hand, in the central region of the magnet, Dy diffusion was
Table 2 Composition of Nd, Dy, and Al of the magnet (a) untreated, treated by (b) the conventional GBD process with DyAl alloy and (c) the present GBD process with Dy-Al co-sorption, measured by ICP.

\begin{tabular}{lccc}
\hline & \multicolumn{3}{c}{ Composition / wt.\% } \\
\cline { 2 - 4 } & $\mathrm{Nd}$ & $\mathrm{Dy}$ & $\mathrm{Al}$ \\
\hline (a) untreated & 31.22 & $<0.01$ & 0.15 \\
\hline (b) DyAl alloy & 30.45 & 1.17 & 0.39 \\
(c) Dy-Al co-sorption & 30.37 & 1.20 & 0.61 \\
\hline
\end{tabular}

clearly observed in the present GBD process with Dy-Al co-sorption, but was slight in the DyAl alloy (see Fig. 5(b) and (c) $1.75 \mathrm{~mm}$-Dy). Although we have reported various GBD processes, ${ }^{7,8,17)}$ the diffusion distance from the magnet surface was several tens of micrometers to several hundreds of micrometers when Dy was applied as a diffusion material. Therefore, notably, the present GBD process exhibited sufficient Dy diffusion up to $1.75 \mathrm{~mm}$ from the magnet 
surface. This is also confirmed by Fig. 5(d), which shows the diffusion distance of Dy from the magnet surface. In the DyAl alloy, the Dy diffusion was pronounced near the surface to approximately $0.1 \mathrm{~mm}$, but the amount of Dy reaching the center region was higher in the $\mathrm{Dy}-\mathrm{Al}$ co-sorption than in the DyAl alloy. Therefore, the diffusivity of Dy in the present GBD process is superior to that of the conventional GBD process. Lu et al. ${ }^{19)}$ reported that the GBD process with $\mathrm{Tb}-$ $\mathrm{Pr}-\mathrm{Cu}-\mathrm{Al}$ alloy applied to the magnet surface improves the wettability and mobility of the diffusion alloy during heat treatment owing to the effect of $\mathrm{Al}$, resulting in high coercivity. In addition, they also suggested that the $\mathrm{Al}$ content of the diffusion alloy reached $25 \mathrm{at} \%, \mathrm{~Tb}$ and $\mathrm{Al}$ diffusion became more pronounced near the magnet surface where it was in direct contact with the diffusion alloy. This is in good agreement with the results of the DyAl alloy containing 50 at\% $\mathrm{Al}$ in this study, that is, a large amount of Dy was consumed by excessive Dy diffusion into the $\mathrm{Nd}_{2} \mathrm{Fe}_{14} \mathrm{~B}$ main phase near the magnet surface, which prevents sufficient Dy diffusion into the center region of the magnet. On the other hand, in the present GBD process, Al was not alloyed with Dy and diffused preferentially compared to Dy. Therefore, the microstructure or chemical properties of the grain boundary is expected to change, but the wettability or mobility of the diffusion material itself should not be directly affected as in the case of DyAl alloy. Thus, in the present GBD process with Dy-Al co-sorption, a deeper Dy diffusion into the center region of the magnet was achieved, resulting in an effective enhancement of the coercivity. In the present GBD magnet, it was confirmed that, in addition to Dy, $\mathrm{Al}$ diffused along the grain boundary to the center region of the magnet compared to the conventional GBD magnet (see Fig. 5(b) and (c) $1.75 \mathrm{~mm}-\mathrm{Al}$ ).

Then, STEM observations were applied to investigate the grain boundary microstructure in more detail. STEM observations were performed around the center region of the magnets. Figure 6 shows STEM high-angle annular-darkfield (HAADF) images of the magnet (a) untreated and (b) treated by the present GBD process with Dy-Al co-sorption. The grain boundary of the untreated magnet was amorphous and thin, but the present GBD magnet was thicker than the untreated magnet and showed a periodic pattern, suggesting the existence of a crystalline phase. The thicknesses of the grain boundary before and after the GBD process were approximately 1.4 and $2.4 \mathrm{~nm}$, respectively. The reason for the thicker grain boundary of the present GBD magnet is that $\mathrm{Nd}$ was substituted by diffused Dy near the surface of the $\mathrm{Nd}_{2} \mathrm{Fe}_{14} \mathrm{~B}$ main phase and precipitated $\mathrm{Nd}$ was enriched in the grain boundary, as previously reported. ${ }^{13,14)}$ Figures $6(\mathrm{c})$ (e) shows the fast Fourier transform (FFT) patterns taken from the grain boundary of the present GBD magnet, and each pattern was obtained from the region indicated by the white circles in Fig. 6(b). The FFT patterns show a discrete ring pattern (denoted by yellow arrows in Fig. 6(c)-(e)), suggesting the existence of an amorphous phase in the grain boundary. Note that, since a beam diameter applied to the FFT was narrowed down to $2 \mathrm{~nm}$, the ring pattern showing an amorphous phase was discrete. This ring pattern was also slightly observed, even at the grain boundary, indicating a symmetric lattice fringe in the STEM image (see Fig. 6(d)).
The FFT patterns in Fig. 6(d) also show a non-ferromagnetic fcc NdO [lll 112$]$ phase with a measured d-space of the (1 1 -1) plane for $0.293 \mathrm{~nm}(0.288 \mathrm{~nm}$ for the International Center for Diffraction Data (ICDD): Card No. 00-033-0937). STEM observations revealed that the grain boundary of the present GBD magnet comprised an fcc $\mathrm{NdO}$ phase and an amorphous phase. To date, many researchers have observed the microstructure of the $\mathrm{Nd}_{2} \mathrm{Fe}_{14} \mathrm{~B}$ grain boundary to obtain guidelines for increasing the coercivity. Commonly reported that the grain boundary contains Nd-rich amorphous phase, ${ }^{14,15,29,30)}$ several $\mathrm{Nd}$ oxide phases, ${ }^{31,32)}$ and $\mathrm{Cu}$-rich phase. ${ }^{14,15,29,30)}$ Watanabe et al. ${ }^{5,6)}$ applied the GBD process with $\mathrm{Tb}$-vapor sorption and reported that the resulting $\mathrm{Nd}$ rich phase consisted mainly of a crystalline $\mathrm{NdO}_{2}$ phase and a minor amorphous phase including $\mathrm{Fe}, \mathrm{Co}$, and $\mathrm{Cu}$, which is in good agreement with the results of this study. Thus, there are various reports on the grain boundary microstructure, and further research is required. Figure 6(f) shows the STEMHAADF image and STEM-EDX elemental mappings for $\mathrm{Nd}$, Dy, $\mathrm{Al}, \mathrm{Fe}, \mathrm{Cu}, \mathrm{Co}$, and $\mathrm{O}$ of the present GBD magnet. The elemental mappings show that $\mathrm{Dy}, \mathrm{Al}, \mathrm{Cu}$, and $\mathrm{Co}$ were also enriched in the Nd-rich grain boundary, while Fe was diluted. The FFT patterns demonstrated the existence of an amorphous phase and an fcc $\mathrm{NdO}$ phase in the grain boundary, and STEM-EDX elemental mapping revealed the enrichment of $\mathrm{Dy}, \mathrm{Al}, \mathrm{Cu}$, and $\mathrm{Co}$, similar to $\mathrm{Nd}$. Combining these results, $\mathrm{Dy}, \mathrm{Al}, \mathrm{Cu}$, and $\mathrm{Co}$ in the grain boundary existed as an amorphous phase.

Figure 7 shows the STEM-HAADF image of the present GBD magnet and the concentration line profile of $\mathrm{Fe}, \mathrm{Nd}, \mathrm{O}$, and $\mathrm{Al} . \mathrm{Co}, \mathrm{Cu}$, and Dy near and at the grain boundary were extracted by STEM-EDX area-analysis. The STEM-EDX was collected in the yellow box shown in Fig. 7(a). The concentration shown in Fig. 7(a) was integrated along the vertical line in the box. Note that, Fig. 6(f) and Fig. 7(a) are for the same region. Compared with the $\mathrm{Nd}_{2} \mathrm{Fe}_{14} \mathrm{~B}$ main phase, the concentration of $\mathrm{Fe}$ in the grain boundary decreased, while the other elements increased, and the concentration of $\mathrm{Nd}$ was 24.9 at\%. For Dy and Al which applied as a diffusion material in the present GBD process, the concentrations increased to $1.3 \mathrm{at} \%$ and 3.4 at $\%$, respectively. While the thickness of the grain boundary of the present GBD magnet was approximately $2.4 \mathrm{~nm}$ (see Fig. 6(b)), the concentration profile shows that the full width at half maximum (FWHM) of Dy and Al was about $4 \mathrm{~nm}$. This indicates that Dy and $\mathrm{Al}$ were enriched beyond the grain boundary to near the surface of the $\mathrm{Nd}_{2} \mathrm{Fe}_{14} \mathrm{~B}$ main phase, which enhances the coercivity but decreases the remanence, as mentioned above. According to a previous study, ${ }^{2)}$ substituting $\mathrm{Nd}$ with $\mathrm{Dy}$ in the $\mathrm{Nd}_{2} \mathrm{Fe}_{14} \mathrm{~B}$ main phase enhances the magnetic anisotropy field $\left(H_{A}\right)$ from 5332 $\mathrm{kA} \cdot \mathrm{m}^{-1}$ to $11937 \mathrm{kA} \cdot \mathrm{m}^{-1}$, but decreases the saturation magnetization $\left(M_{s}\right)$ from $1.60 \mathrm{~T}$ to $0.71 \mathrm{~T}$. Assuming that the $(\mathrm{Nd}, \mathrm{Dy})_{2} \mathrm{Fe}_{14} \mathrm{~B}$ shell comprises $\mathrm{Nd}$ and $\mathrm{Dy}$ in the ratio of $24.9: 1.3 \mathrm{at} \%$, its magnetic anisotropy is estimated at $5660 \mathrm{kA} \cdot \mathrm{m}^{-1}$, which is an increase of $6.1 \%$, and its saturation magnetization is estimated at $1.56 \mathrm{~T}$, which is a decrease of $2.5 \%$. Note that, this estimation is based on the assumption that the composition of the $\mathrm{Nd}_{2} \mathrm{Fe}_{14} \mathrm{~B}$ main phase core is Dy-free $\mathrm{Nd}_{2} \mathrm{Fe}_{14} \mathrm{~B}$. However, the actual increase ratio in the 


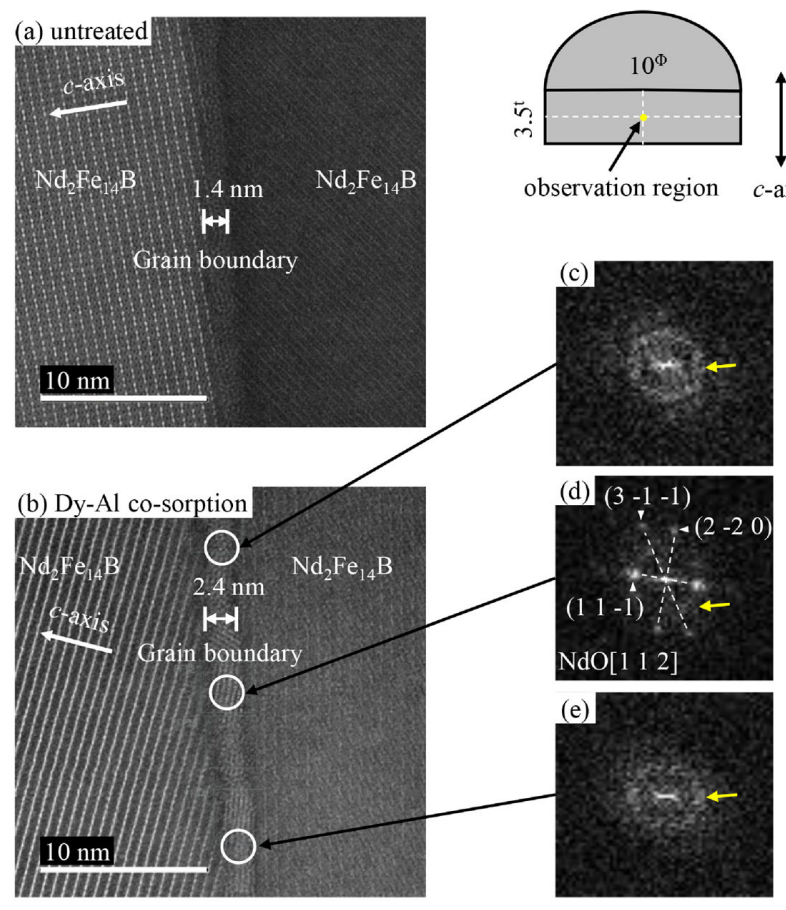

(f)
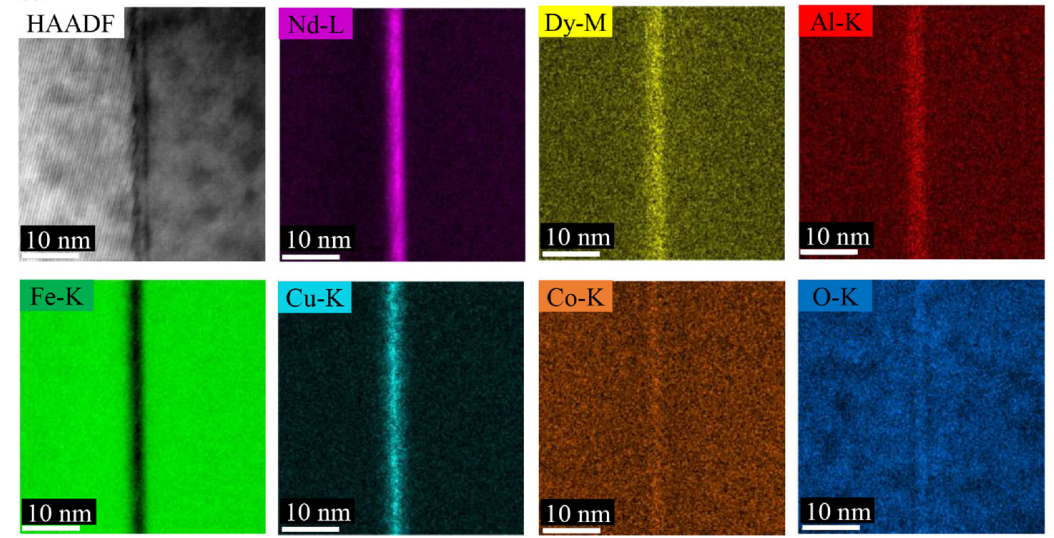

Fig. 6 STEM high-angle-annular-dark-field (HAADF) images of the magnet (a) untreated and (b) treated by the present GBD process with Dy-Al co-sorption. (c)-(e) Fast Fourier transform (FFT) patterns taken from the grain boundary of the present GBD magnet. Each pattern is obtained from the region indicated by the white circles in (b) and (f) STEM-HAADF image and STEM-EDX elemental mappings for $\mathrm{Nd}$, $\mathrm{Dy}, \mathrm{Al}, \mathrm{Fe}, \mathrm{Cu}, \mathrm{Co}$, and $\mathrm{O}$ of the present GBD magnet.

coercivity was much higher than the estimated increase ratio in the magnetic anisotropy field. This may be attributed to the difference in Dy concentration between the surface and center regions of the magnet, particularly because the center region where the estimation was applied, the Dy concentration was lower compared to the surface region. In addition, it is also considered that there are other factors, such as changing the grain boundary microstructure, which contributes to the increase in coercivity. Meanwhile, the actual decrease ratio in the remanence was slightly higher than the estimated decrease ratio in the saturation magnetization. The saturation magnetization reportedly decreased due to the substitution of $\mathrm{Fe}$ by $\mathrm{Al}$ at the $j_{2}$ site of the $\mathrm{Nd}_{2} \mathrm{Fe}_{14} \mathrm{~B}$ phase, ${ }^{33)}$ this may be one reason for the present result.

Then, we discuss the role of $\mathrm{Al}$, which is an important element in this study. Oono et al. ${ }^{18)}$ suggested that the existence of diffused elements such as $\mathrm{Ni}$ and $\mathrm{Al}$ in the grain boundary may contribute to the deep diffusion of Dy by decreasing the melting point of the grain boundary. Combining the experimental results, that is, microstructure observation and concentration profiling, with this speculation, the melting point of the grain boundary is expected to decrease by approximately $430 \mathrm{~K}$ in the phase diagram of the $\mathrm{Nd}-\mathrm{Al}$ binary system. ${ }^{25)}$ As a marked decrease in the melting point of the grain boundary, the diffusivity of Dy improved during the GBD process by actively transforming the grain boundary into the liquid-state grain boundary. Hence, a deeper Dy diffusion was achieved, and the coercivity was effectively enhanced.

\subsection{Thermal demagnetization and magnetic domain observation}

Figure 8 shows the coercivity measured at $300 \mathrm{~K}, 373 \mathrm{~K}$, and $453 \mathrm{~K}$ for the magnet (a) treated by the present GBD process with Dy-Al co-sorption and (b) untreated, evaluated by SCM-VSM. The coercivity of the present GBD magnet at 
(a)

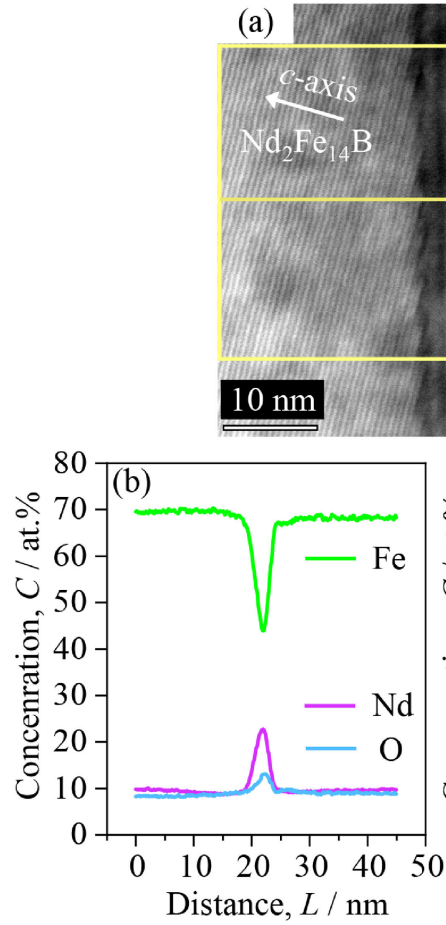

$\mathrm{Nd}_{2} \mathrm{Fe}_{14} \mathrm{~B}$

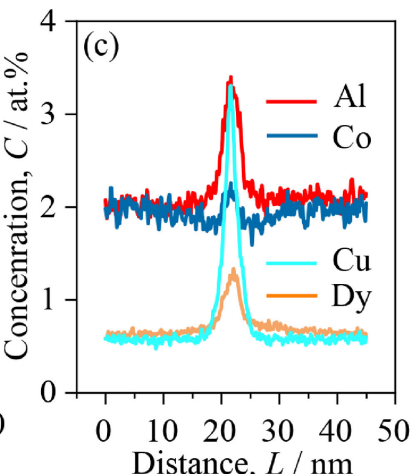

Fig. 7 STEM-HAADF image of the present GBD magnet (a) and concentration line profile of (b) $\mathrm{Fe}, \mathrm{Nd}, \mathrm{O}$ and (c) $\mathrm{Al}, \mathrm{Co}, \mathrm{Cu}$, and $\mathrm{Dy}$ near and the grain boundary extracted by STEM-EDX area-analysis. The STEM-EDX was collected in the yellow box shown in (a) and the concentration was integrated along the vertical line in the box.

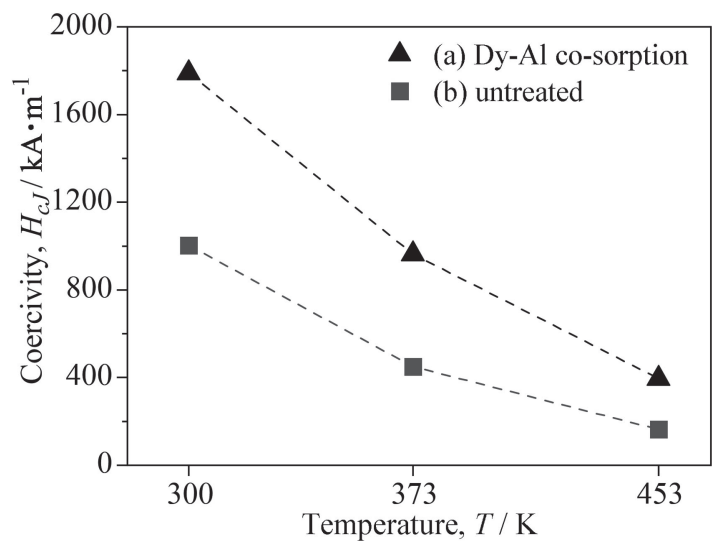

Fig. 8 Coercivity measured at $300 \mathrm{~K}, 373 \mathrm{~K}$, and $453 \mathrm{~K}$ for the magnet (a) treated by the present GBD process with $\mathrm{Dy}-\mathrm{Al}$ co-sorption and (b) untreated.

$373 \mathrm{~K}$ was similar to that of the untreated magnet at $300 \mathrm{~K}$. In addition, the temperature coefficient of coercivity $\beta$ also improved from $-0.53 \% \cdot \mathrm{K}^{-1}$ to $-0.50 \% \cdot \mathrm{K}^{-1}$, indicating that the present GBD magnet improved the heat resistance in accordance with the increase in coercivity.

To study the behavior of thermal demagnetization in more detail, magnetic domain observations using MFM were performed. The MFM observations were performed on a polished surface perpendicular to the $c$-axis, and the polishing depth from the surface was approximately $100 \mu \mathrm{m}$. In these observation regions, the magnetic anisotropy field was effectively enhanced by the GBD process. The MFM images for the untreated magnet at each observation temperature are shown in Fig. 9(a) and schemati-

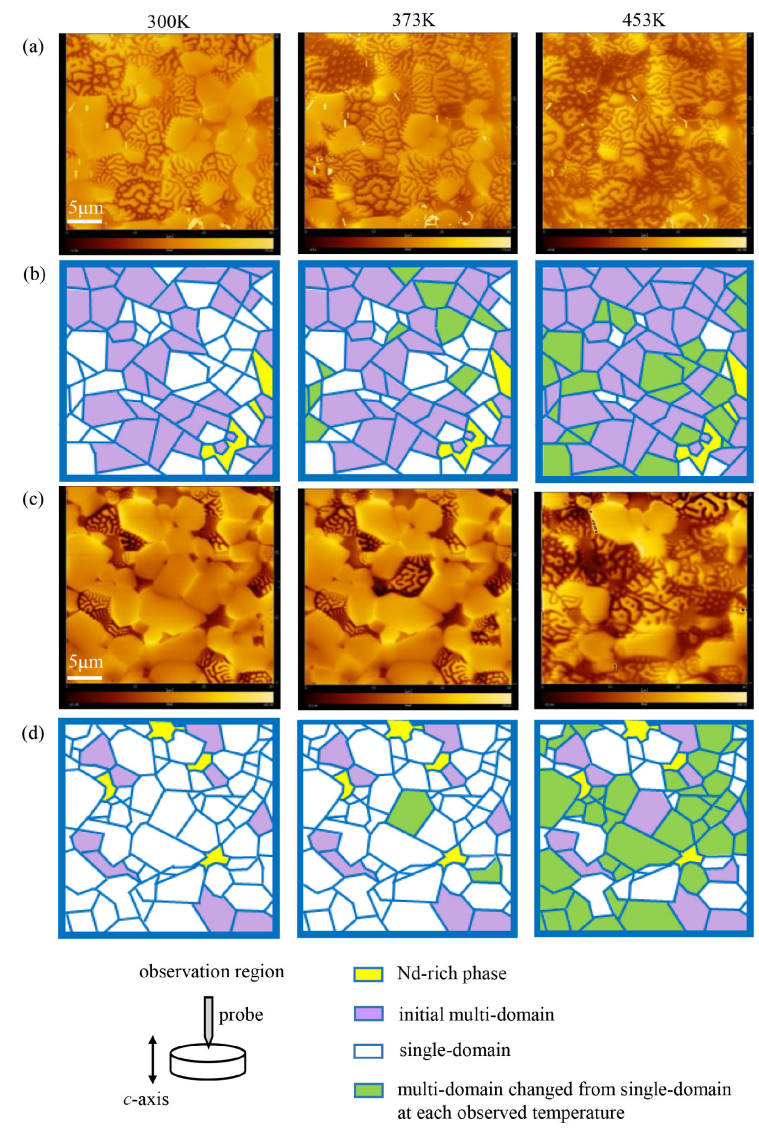

Fig. 9 MFM images and schematic drawings obtained at $300 \mathrm{~K}, 373 \mathrm{~K}$ and $453 \mathrm{~K}$ for the thermal demagnetized magnet (a), (b) untreated and (c), (d) treated by the present GBD process with Dy-Al co-sorption.

Table 3 Demagnetization ratio of the magnet (a) untreated and (b) treated by the present GBD process with Dy-Al co-sorption at each temperature.

Demagnetization ratio

$(\%)$

\begin{tabular}{ccc}
\hline $\begin{array}{c}\text { Temperature } \\
\text { / K }\end{array}$ & (a) untreated & (b) Dy-Al co-sorption \\
\hline 300 & 47.4 & 11.1 \\
373 & 60.3 & 13.6 \\
453 & 89.7 & 61.7 \\
\hline
\end{tabular}

cally drawn in Fig. 9(b). In the schematic, the magnetic domain state of each grain is shown in each color. The MFM images and schematic drawings of the present GBD magnet obtained by the same method are shown in Fig. 9(c) and 9(d). The demagnetization ratios at each observed temperature are summarized in Table 3. The demagnetization ratio is defined as follows:

Demagnetization ratio $(\%)$

$$
=\frac{\text { the number of multi-domain grains }}{\text { the number of total grains }- \text { Nd-rich phase }} \times 100
$$

At $300 \mathrm{~K}$, despite being magnetized at $6366 \mathrm{kA} \cdot \mathrm{m}^{-1}$, a significant number of multi-domain grains with maze patterns were observed. The existence of such multiple domains in the initial state may be due to the existence of grains with almost zero coercivity, which are caused by a local decrease in the 
magnetic anisotropy field by polishing the magnet surface, as reported by Kobayashi et al. ${ }^{34)}$ The demagnetization ratios at $300 \mathrm{~K}$ were $47.4 \%$ and $11.1 \%$ in the untreated and present GBD magnets. In the untreated magnet, the adjacent grains appeared to have a continuous multi-domain structure, whereas in the present GBD magnet, the multi-domain structure did not exhibit such strong continuity; rather, they appear to be isolated. This may be because the nonferromagnetic fcc $\mathrm{NdO}$ phase in the present GBD magnet existed in the grain boundary, which contributed to the magnetic decoupling between the $\mathrm{Nd}_{2} \mathrm{Fe}_{14} \mathrm{~B}$ main phases, and also enhanced the magnetic anisotropy field by enriching Dy at the surface of the $\mathrm{Nd}_{2} \mathrm{Fe}_{14} \mathrm{~B}$ main phase. The surface defects of the $\mathrm{Nd}_{2} \mathrm{Fe}_{14} \mathrm{~B}$ main phase, that is, magnetically coupled grains, and the high local demagnetization stray field at the sharp corners and edges of grains, are reportedly the cause of the decrease in the magnetic anisotropy field. ${ }^{35-38)}$ In the present GBD magnet, such surface defects in the $\mathrm{Nd}_{2} \mathrm{Fe}_{14} \mathrm{~B}$ main phase were repaired by the formation of a thick grain boundary and a high magnetic anisotropy field $(\mathrm{Nd}, \mathrm{Dy})_{2} \mathrm{Fe}_{14} \mathrm{~B}$ shells, thereby suppressing magnetization reversal. Bance et al. ${ }^{39)}$ reported that $(\mathrm{Dy}, \mathrm{Nd})_{2} \mathrm{Fe}_{14} \mathrm{~B}$ shells with a thickness of $4 \mathrm{~nm}$ around the $\mathrm{Nd}_{2} \mathrm{Fe}_{14} \mathrm{~B}$ main phase canceled out the reduction of the coercivity owing to the existence of surface defects. The thickness of these shells are in good agreement with the approximately $4 \mathrm{~nm}$ FWHM of the Dy concentration profile confirmed by the present GBD magnet. (see Fig. 7(c)) In fact, in the present GBD magnet with high coercivity, most of the magnetic domains persisted the single-domain state at $373 \mathrm{~K}$, but the untreated magnet demagnetized by $60.3 \%$. Finally, at $453 \mathrm{~K}, 89.7 \%$ of the untreated magnet and $61.7 \%$ of the present GBD magnets were found to have a multi-domain structure due to thermal demagnetization. Although these results show a similar trend to the thermal degradation of coercivity at the same temperature shown in Fig. 8, there was a difference of approximately $10 \%$ in the absolute value of the demagnetization ratio. This may be because the comparison is based on the total coercivity measured as the magnets and the results of the magnetic domain observations on a local surface region that is susceptible to demagnetization.

\section{Summary}

We applied an effective GBD process with Dy-Al cosorption to $\mathrm{Nd}-\mathrm{Fe}-\mathrm{B}$ sintered magnets. The coercivity of the present GBD magnet increased from $1003 \mathrm{kA} \cdot \mathrm{m}^{-1}$ to $1789 \mathrm{kA} \cdot \mathrm{m}^{-1}$. This increase in coercivity is higher than that of conventional GBD magnets treated with DyAl alloy $\left(H_{c J}=1661 \mathrm{kA} \cdot \mathrm{m}^{-1}\right) \quad$ and $\quad \mathrm{TbF}_{3}+\mathrm{CaH}_{2} \quad\left(H_{c J}=1761\right.$ $\left.\mathrm{kA} \cdot \mathrm{m}^{-1}\right)$. The present GBD process facilitated Dy diffusion into the center region of the magnet with a thickness of $3.5 \mathrm{~mm}$, resulting in high coercivity. This is presumably because the preferential Al diffusion decreased the melting point of the grain boundary, thereby improving the diffusivity of Dy. STEM observations revealed that Dy and Al were clearly enriched in the grain boundary, even in the center region of the magnet. Furthermore, it was also demonstrated that the grain boundary comprised of an fcc $\mathrm{NdO}$ phase and an amorphous phase including Dy, Al, Cu, and Co. As an extension study, the thermal demagnetization behavior was observed using MFM. In the present GBD magnet, the existence of a non-ferromagnetic fcc $\mathrm{NdO}$ phase, which contributes to magnetic decoupling between the $\mathrm{Nd}_{2} \mathrm{Fe}_{14} \mathrm{~B}$ main phases, and the formation of $(\mathrm{Dy}, \mathrm{Nd})_{2} \mathrm{Fe}_{14} \mathrm{~B}$ shells with a high magnetic anisotropy field suppressed the continuous domain reversal of adjacent grains. As a result, in the present GBD magnet, a partially persistent single-domain structure was observed, even at $453 \mathrm{~K}$.

\section{Acknowledgements}

The authors would like to thank Tamakawa Co., Ltd. and Nitto Analysis Center Co., Ltd. for providing the SCM-VSM and MFM, respectively.

\section{REFERENCES}

1) N.C. Koon, B.N. Das, M. Rubinstein and J. Tyson: J. Appl. Phys. 57 (1985) 4091-4093.

2) S. Hirosawa, Y. Matsuura, H. Yamamoto, S. Fujimura and M. Sagawa: J. Appl. Phys. 59 (1986) 873-879.

3) M. Sagawa, S. Hirosawa, K. Tokuhara, H. Yamamoto and S. Fujiwara: J. Appl. Phys. 61 (1987) 3559.

4) Y. Matsuura: J. Magn. Magn. Mater. 303 (2006) 344-347.

5) N. Watanabe, H. Umemoto, M. Ishimaru, M. Itakura, M. Nishida and K. Machida: J. Microscopy 236 (2009) 104-108.

6) N. Watanabe, H. Umemoto, M. Itakura, M. Nishida and K. Machida: J. Phys. IOP Conf. Ser.: Mater. Sci. Eng. 1 (2009) 012033.

7) D. Li, S. Suzuki, T. Kawasaki and K. Machida: Jpn. J. Appl. Phys. 47 (2008) 7876-7878

8) D. Li, S. Suzuki, M. Suzuki, S. Nishiyama, M. Itoh and K. Machida: IOP Conf. Ser. Mater. Sci. Eng. 1 (2009) 012020.

9) T. Horikawa, M. Itoh, S. Suzuki and K. Machida: J. Magn. Magn. Mater. 271 (2004) 369-380.

10) H. Nakamura, K. Hirota, M. Shimano, T. Minowa and M. Honshima: IEEE Trans. Magn. 41 (2005) 3844-3846.

11) K. Hirota, H. Nakamura, T. Minowa and M. Honshima: IEEE Trans. Magn. 42 (2006) 2909-2911

12) H. Nakamura, K. Hirota, T. Minowa and M. Honshima: J. Magn. Soc. Jpn. 31 (2007) 6-11 (in Japanese).

13) M. Soderžnik, K.Ž. Rožman, S. Kobe and P. McGuiness: Intermetallics 23 (2012) 158-162.

14) H. Sepehri-Amin, T. Ohkubo and K. Hono: J. Appl. Phys. 107 (2010) 09A745.

15) H. Sepehri-Amin, T. Ohkubo and K. Hono: Acta Mater. 61 (2013) 1982-1990.

16) T. Odaka, H. Morimoto, K. Yoshimura, S. Takaki and S. Sakashita: Japan Patent, (2009) 016815.

17) M. Uenohara, Z. Hanlng, H. Nishio and K. Machida: Mater. Trans. 61 (2020) 782-786.

18) N. Oono, M. Sagawa, R. Kasada, H. Matsui and A. Kimura: J. Magn. Magn. Mater. 323 (2011) 297-300.

19) K. Lu, X. Bao, Y. Zhou, X. Lv, Y. Ding, M. Zhang, C. Wang and X. Gao: J. Magn. Magn. Mater. 500 (2020) 166384.

20) N.E. Topp: Chemistry of the Rare-Earth Elements, (Elsevier Publishing Company, Amsterdam, 1965) pp. 156-157.

21) Handbook of Chemistry: Pure Chemistry, 5th ed., vol. 1, (The Chemical Society of Japan, 2004) p. 30.

22) C.E. Habermann and A.H. Daane: J. Chem. Phys. 41 (1964) 2818 2827.

23) S. Pandian and V. Chandrasekaran: J. Appl. Phys. 92 (2002) 60826086.

24) J.J. Ni, T.Y. Ma, Y.R. Wu and M. Yan: J. Magn. Magn. Mater. 322 (2010) 3710-3713

25) K.H.J. Buschow: J. Less-Common Met. 9 (1965) 452-456.

26) H. Nishio, K. Machida and K. Ozaki: IEEE Trans. Magn. 53 (2017) 6000306. 
27) H. Nishio and K. Machida: IEEE Trans. Magn. 54 (2018) 6000904

28) T. Fukagawa and S. Hirosawa: J. Appl. Phys. 104 (2008) 013911

29) W.F. Li, T. Ohkubo and K. Hono: Acta Mater. 57 (2009) 1337-1346.

30) H. Sepehri-Amin, T. Ohkubo, T. Shima and K. Hono: Acta Mater. 60 (2012) 819-830.

31) W. Mo, L. Zhang, Q. Liu, A. Shan, J. Wu and M. Komuro: Scr. Mater 59 (2008) 179-182.

32) T.G. Woodcock and O. Gutfleisch: Acta Mater. 59 (2011) 1026-1036.

33) Y.C. Yang, D.E. Tharp, G.J. Long, O.A. Pringle and W.J. James: J. Appl. Phys. 61 (1987) 4343-4345.

34) K. Kobayashi, K. Itoh, D. Shimizu and K. Hayakawa: J. Magn. Soc. Jpn. 31 (2007) 393-397.
35) J.F. Herbst: Rev. Mod. Phys. 63 (1991) 819-898.

36) H. Kronmüller and K.D. Durst: J. Magn. Magn. Mater. 74 (1988) 291302.

37) R. Goto, M. Matsuura and S. Sugimoto: J. Appl. Phys. 111 (2012) 07A739.

38) S. Bance, H. Oezelt, T. Schrefl, G. Ciuta, N.M. Dempsey, D. Givord, M. Winklhofer, G. Hrkac, G. Zimanyi, O. Gutfleisch, T.G. Woodcock, T. Shoji, M. Yano, A. Kato and A. Manabe: Appl. Phys. Lett. 104 (2014) 182408.

39) S. Bance, J. Fischbacher and T. Schrefl: J. Appl. Phys. 117 (2015) 17A733. 